\title{
Electron Diffraction-Based Quality Evaluation of Graphene Films
}

\author{
Kai Cui ${ }^{1}$, Ken Bosnick ${ }^{1}$, Rob Indoe ${ }^{1}$, and Marek Malac ${ }^{1,2}$ \\ 1. National Institute for Nanotechnology, Edmonton, Canada \\ 2. Department of Physics, University of Alberta, Edmonton, Canada
}

Graphene appears to be a material with many potential applications. A robust and convenient method is therefore needed to evaluate the quality of graphene films. Raman spectroscopy is often used and is fast and inexpensive; however, the information content in the Raman spectrum is limited. We demonstrate an electron diffraction based method that can be routinely used to evaluate the quality of graphene thin films.

Graphene membranes were fabricated by transferring CVD grown graphene on to holey silicon nitride membranes [1]. TEM imaging and diffraction was performed using a Hitachi H-9500 environmental TEM operated at $100 \mathrm{kV}$. A typical low magnification image and select area electron diffraction pattern (DP) are shown in Fig. $1 \mathrm{a}$ ) and b). For analysis, the DPs were rotationally averaged using the DiffTools package [2] in Gatan Digital Micrograph. A sample rotationally averaged diffractogram is shown in Fig. 1 c) with peaks at $4.7\{100\}, 8.1\{110\}$, and $9.4 \mathrm{~nm}^{-1}\{200\}$ [3]. After a background subtraction fit to regions beside the peak of interest, single or double Lorentzians were fit to the peak as shown in Fig. 1 d) and e). In order to perturb the quality of the graphene, the membrane was heated in the TEM from room temperature to $950^{\circ} \mathrm{C}$, with images and DPs collected in $50^{\circ} \mathrm{C}$ intervals. The membranes seldom tear during the heat treatment. However, a shoulder begins to appear on the high spatial frequency (smaller lattice spacing) side of the $\{100\}$ and $\{110\}$ peaks in DPs at $\mathrm{T}_{\mathrm{C}}$ exceeding 150 to $350{ }^{\circ} \mathrm{C}$, depending on the sample. The peaks are well fit with single Lorentzian below $\mathrm{T}_{\mathrm{C}}$ and with double Lorentzian above it. Inspection of the corresponding diffraction patterns in Fig. 2 a) reveals that the shoulder does not have the six-fold symmetry of the $\{100\}$ peaks but is a ring close to the first order diffraction peak of graphene. The shoulder center at $5.1 \mathrm{~nm}^{-1}$ does not change with increasing temperature, as shown by the double Lorentzian fitting parameters in Fig. 2 c). Control experiments confirm that the growth of the shoulder is due to heating and that electron beam irradiation has no noticeable effect. Moreover, heating experiments in the presence of $\mathrm{H} 2$ at $10^{-4} \mathrm{~Pa}$ confirm that the diffraction ring is not a result of graphene oxide formation [3]. Cooling the membrane to room temperature does not affect the observed DPs. The results are consistent with the formation of disordered centers with lattice spacing slightly smaller than graphene. One possible explanation is the rearrangement of six-membered graphene rings to five-membered rings [4,5]. The ratio of the crystalline and disordered components is evaluated by the intensity ratio of the six diffraction spots to the diffraction ring obtained using the arc function in DiffTools [2], as shown in Fig. 2 b). The ratio decreases when heating from room temperature to $\mathrm{T}_{\mathrm{C}}$ and becomes constant above $\mathrm{T}_{\mathrm{C}}$, as shown in Fig. $2 \mathrm{~d}$ ). Extensive hold time at $950^{\circ} \mathrm{C}$ does not further affect the ratio. Since both the spots and rings arise from carbon with known scattering factor $f(q)$ quantitative evaluation of the volume ratio of disordered and ordered components is possible. In conclusion, a fast and convenient method to evaluate the quality of graphene membranes by means of standard selected area electron diffraction was demonstrated.

\section{References:}

[1] JW Suk et al, ACS Nano 5 (2011), p. 6916. 
[2] DRG Mitchell, Micros Res Tech 71 (2008), p. 588.

[3] EC Mattson et al, ACS Nano 5 (2011), p. 9710.

[4] A Chuvillin et al, New J Phys 11 (2009), p. 083019.

[5] C Jin et al, Phys Rev Lett 102 (2009), p. 205501.
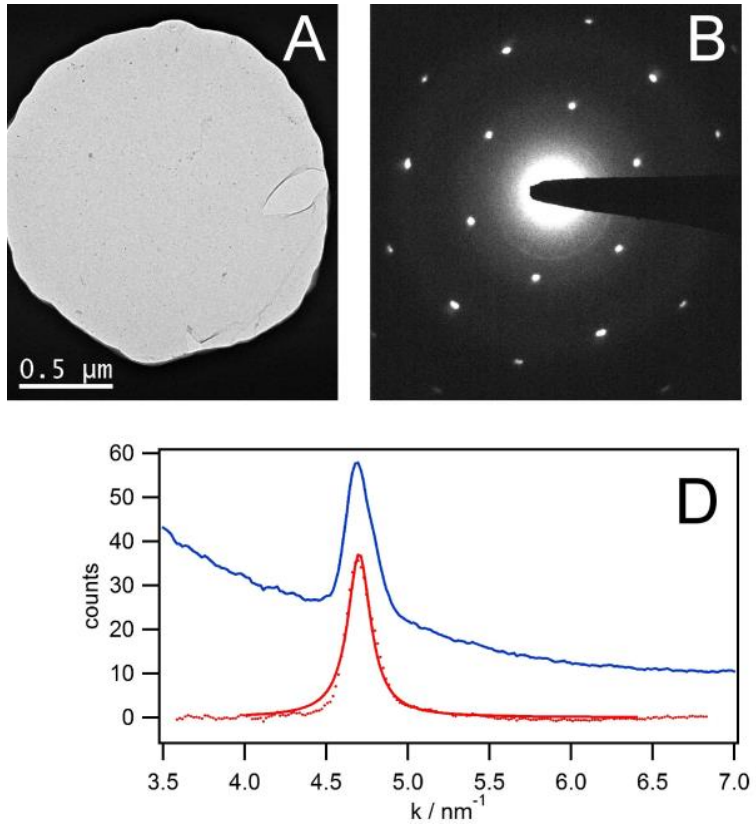
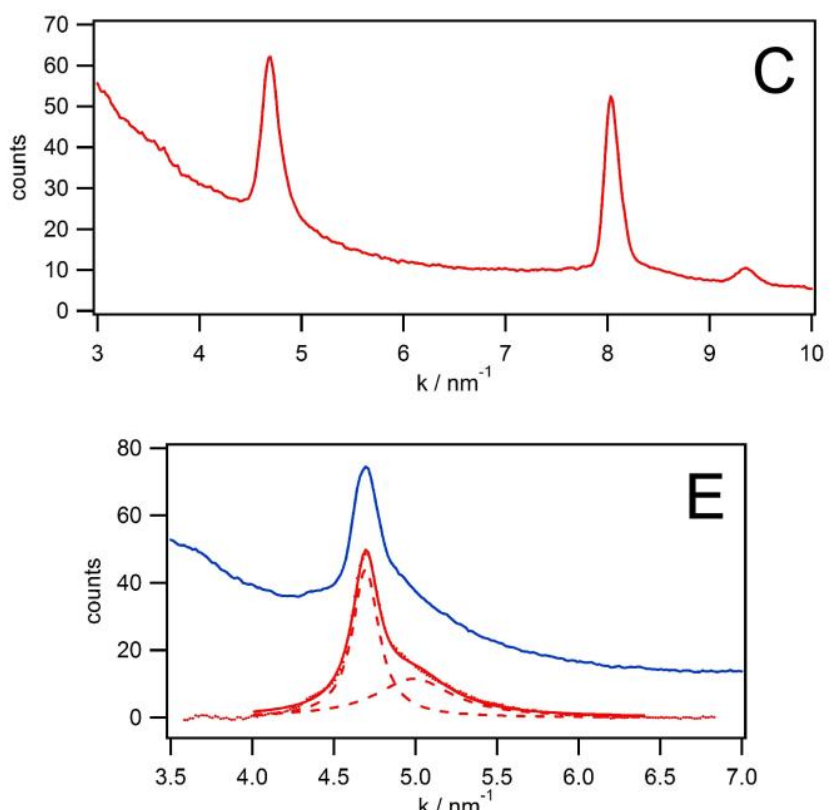

Figure 1. (A) TEM image and (B) electron diffraction pattern from a graphene membrane. (C) Rotationally averaged diffraction pattern. (D,E) Fits (red) to the background subtracted (dots), rotationally averaged diffraction pattern (blue) in the $\{100\}$ region: (D) single Lorentzian fit, $100^{\circ} \mathrm{C},(\mathrm{E})$ double Lorentzian fit, $900^{\circ} \mathrm{C}$.
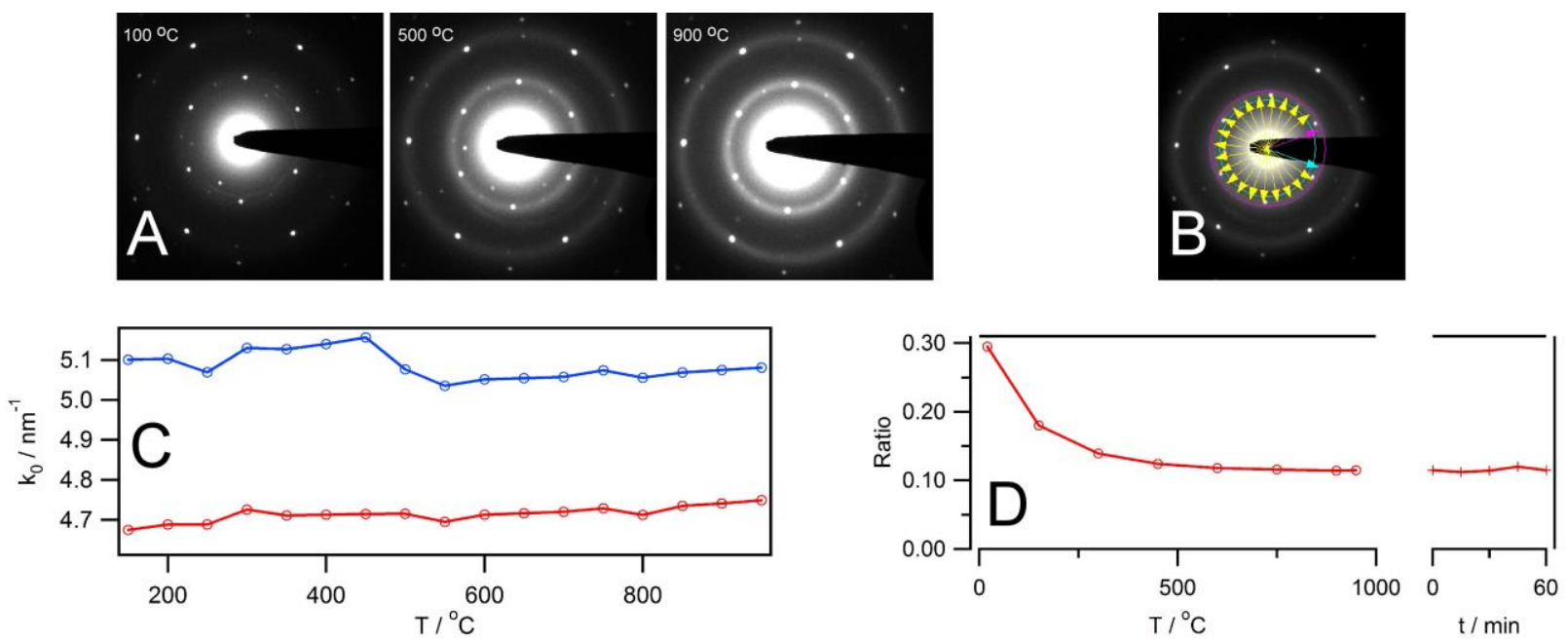

Figure 2. (A) Diffraction patterns at different temperatures; (B) arc function evaluation of spot to ring intensity ratio; (C) peak center variation with temperature from double Lorenzian fits, and (D) spot to ring ratio with temperature (left) and hold time at $950{ }^{\circ} \mathrm{C}$ (right). 